\title{
Characteristics of Oil Palm EFB (Empty Fruit Bunch of Elaeis guineensis) Lignin
}

\author{
Takanori Shinano $^{1}$, Masamitsu Funaoka ${ }^{1}$, Yoshihito Shirai ${ }^{2}$ and Mohd Ali Hassan ${ }^{3}$ \\ ${ }^{1}$ Graduate school of Bioresources, Mie University, SORST JST \\ ${ }^{2}$ Kyushuu Institute of Technology \\ ${ }^{3}$ Universiti Putra Malaysia \\ *Fax: 059-231-9517, e-mail: funaoka@bio.mie-u.ac.jp
}

In order to utilize EFB lignin successively following the ecosystem, the characteristics of EFB lignin were discussed in detail. EFB lignophenol was synthesized through the phase-separation system. The pyrogram of EFB lignophenol shows the peaks of phenol, $p$-cresol, guaiacol and syringol. The FT-IR spectrum of EFB lignophenol shows a peak at $1714 \mathrm{~cm}^{-1}$ assigned to esters. EFB lignophenol was subjected to mild alkaline treatment to give guaiacyl - syringyl lignins and p-hydroxybenzoic acid. The thermal stabilities of EFB lignophenol after mild alkaline treatment were increased. GC/MS analysis and above results showed that EFB lignophenol includes esterified $p$-hydroxybenzoic acid. The amounts of attached $p$-hydroxybenzoic acid were $9.6 \%$ of EFB lignophenol $\left(0.23 \mathrm{~mol} / \mathrm{C}_{9}\right.$ in EFB lignophenol) and $1.8 \%$ of EFB extractives-free meals $\left(0.15 \mathrm{~mol} / \mathrm{C}_{9}\right.$ in klason lignin). The difference in frequencies of $p$-hydroxybenzoic acid suggests the heterogeneous incorporation of $p$-hydroxybenzoic acid into lignin.

Key words: Oil Palm, EFB (Empty Fruit Bunch), Phase-Separation System, Lignophenol, Resource Circulation

\section{INTRODUCTION}

Oil Palm EFB (Empty Fruit Bunch of Elaeis guineensis) is one of byproducts from oil palm industry. EFB composed of cellulose, hemicellulose and lignin has been utilized only as fuels and mulches. In the previous work [1], the potential of EFB as aromatic and aliphatic resources through the phase-separation system $[2,3]$ has been discussed. In order to successively utilize EFB from composite to molecular levels, following the carbon cycle in the ecosystem, detailed molecular information of EFB lignin is required. In this work, the characteristics of EFB core lignin and attached units were investigated.

\section{EXPERIMENTAL}

2.1 Synthesis of EFB lignophenol

EFB lignophenol was synthesized by 2 step process II of phase-separation system composed of $p$-cresol and $72 \% \mathrm{H}_{2} \mathrm{SO}_{4}$. In order to solvate lignin, extractives-free EFB meals $(50 \mathrm{~g})$ were covered with acetone solution containing $3 \mathrm{~mol} / \mathrm{C}_{9}$ of $p$-cresol uniformity. After $24 \mathrm{hrs}$, acetone was removed at room temperature. Seventy-two $\% \mathrm{H}_{2} \mathrm{SO}_{4}(500 \mathrm{~mL})$ was added to cresol solvated EFB meals, and stirred vigorously for $60 \mathrm{~min}$. The reaction mixture was added to excess amount of deionized water. The precipitates (lignophenol - carbohydrate complex) were collected by centrifugation, washed up to $\mathrm{pH}$ 6-7 and freeze-dried. EFB lignophenol was extracted with acetone from the resulting composites. The combined lignophenol acetone solution was concentrated, and added dropwise to excess amount of diethyl ether with vigorous stirring. The precipitate (EFB lignophenol) was collected by centrifugation and dried over $\mathrm{P}_{2} \mathrm{O}_{5}$ after evaporating the solvent.

2.2 Preparation of EFB core-lignophenol and determination of $p$-hydroxyphenyl units.

EFB lignophenol $(150 \mathrm{mg})$ was dissolved in $60 \mathrm{~mL}$ of $1 \mathrm{~N} \mathrm{NaOH}$ and stirred under $\mathrm{N}_{2}$ flow at $40^{\circ} \mathrm{C}$ for $72 \mathrm{hrs}$. The solution was acidified up to $\mathrm{pH} 4$ with $1 \mathrm{~N} \mathrm{HCl}$. The precipitate (EFB core-lignophenol) was collected by centrifugation. EFB core-lignophenol was washed with

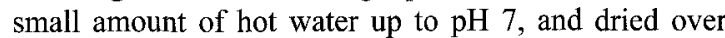
$\mathrm{P}_{2} \mathrm{O}_{5}$. The water soluble part was saturated with $\mathrm{NaCl}$ and extracted with diethyl ether [4]. The ether soluble part was dried over anhydrous $\mathrm{Na}_{2} \mathrm{SO}_{4}$, concentrated, trimethylsilylated and analyzed by GC/MS [a Shimadzu GC/MS-QP 5050A, column: 007-1 (Quadrex, $50 \mathrm{~m} \times$ $0.25 \mathrm{~mm} \times 0.25 \mu \mathrm{m}$ ), carrier gas: He].

In case of quantitative analysis, $\beta$-naphthol was used as an internal standard. The ether soluble part was analyzed by gas chromatography [GC-17A with flame ionization detector, column: 007-1 (Quadrex, $50 \mathrm{~m} \times$ $0.25 \mathrm{~mm} \times 0.25 \mu \mathrm{m})$, carrier gas: He]

2.3 Determination of $p$-hydroxyphenyl units in EFB meals

Extractives-free EFB meals $(1 \mathrm{~g})$ were treated with 1 $\mathrm{N} \mathrm{NaOH}$ under $\mathrm{N}_{2}$ flow at $40^{\circ} \mathrm{C}$ for $96 \mathrm{hrs}$. The insoluble materials were collected by centrifugation and washed with hot water up to $\mathrm{pH} 7$. The alkaline soluble part was acidified with $1 \mathrm{~N} \mathrm{HCl}$, saturated with $\mathrm{NaCl}$ and extracted with diethyl ether. After drying with anhydrous $\mathrm{Na}_{2} \mathrm{SO}_{4}$ and filtration, the ether soluble part was concentrated, trimethylsilylated and analyzed by GC/MS. In case of quantitative analysis, $\beta$-naphthol was used as an internal standard.

2.4 Analyses of EFB lignophenol and EFB core-lignophenol

FT-IR spectra ( $\mathrm{KBr}$ disks) of lignophenols were determined on Spectrum TM GX FT-IR Spectroscopy (PERKIN ELMER). In order to measure molecular 
weight, gel permeation chromatograms of lignophenols were determined on LC-10 system with four columns (KF801, KF802, KF803 and KF804) and UV detector $(280 \mathrm{~nm})$. Tetrahydrofuran was used as the eluent. In order to estimate grafted $p$-cresol contents, ${ }^{1} \mathrm{H}-\mathrm{NMR}$ spectra of original and acetylated lignophenol were determined in $\mathrm{C}_{5} \mathrm{D}_{5} \mathrm{~N}-\mathrm{CDCl}_{3}(1 / 3, \mathrm{v} / \mathrm{v})$ and $\mathrm{CDCl}_{3}$ on a JNM-A500 FT-NMR SYSTEM and ALPHA FT-NMR Spectrometer (JEOL, $500 \mathrm{MHz}$ ). UV-Vis (in methyl cellosolve) spectra of lignophenols were determined by a JASCO V-560 spectrophotometer. TMA profiles of lignophenols were determined by a Seiko Instruments Inc TMA / SS6100 (Rate: $2^{\circ} \mathrm{C} / \min , 50{ }^{\circ} \mathrm{C}-250^{\circ} \mathrm{C}$, Flow: $\mathrm{N}_{2}$ ). TG profiles of lignophenols were determined by a Seiko Instruments Inc TG / DTA 6200 (Rate: $2^{\circ} \mathrm{C} / \min , 50{ }^{\circ} \mathrm{C}-450^{\circ} \mathrm{C}$, Flow: $\mathrm{N}_{2}$ ). In order to estimate compositions of lignophenols, lignophenols were pyrolyzed at $500^{\circ} \mathrm{C}$ for $12 \mathrm{~s}$, using a pyrolyzer (Frontier Lab., PY-2020D) coupled to a gas chromatograph with EI-MS detector (Shimadzu GC/MS-QP5050A, Carrier gas: $\mathrm{He}$ ). The gas chromatographs were fitted with an $007-1$ column programmed from 90 to $280^{\circ} \mathrm{C}$ at $4^{\circ} \mathrm{C}$ /min.

2.6 Switching function of lignophenol

Lignophenols $(5 \mathrm{mg})$ were dissolved in $0.5 \mathrm{~N} \mathrm{NaOH}$ $\left(2 \mathrm{~mL}\right.$ ) and treated at $120{ }^{\circ} \mathrm{C}, 140{ }^{\circ} \mathrm{C}$ and $170{ }^{\circ} \mathrm{C}$. After reactions, the reaction mixture was acidified with $1 \mathrm{~N} \mathrm{HCl}$ to $\mathrm{pH} 2$. The precipitations were collected by centrifugation, washed with small amount of water and dried under $\mathrm{P}_{2} \mathrm{O}_{5}$. The average molecular weights of lignophenols (2nd derivatives) were estimated by GPC.

\section{RESULT AND DISCUSSION}

3.1 Structural features of EFB core-lignophenol

The yields of EFB lignophenol and EFB core-lignophenol were $65.8 \%$ of EFB lignin and $80.3 \%$ of EFB lignophenol, respectively. FT-IR spectrum of EFB core-lignophenol does not have a peak at $1714 \mathrm{~cm}^{-1}$ assigned to ester linkages. This shows ester linkages in EFB lignophenol were hydrolyzed. The spectrum was similar to that of hardwood lignophenol (Fig. 1). Furthermore a peak of phenol to $p$-cresol in pyrogram of EFB core-lignophenol was decreased (Fig. 2). The peak ratio [guaiacol / syringol (area / area)] of EFB lignophenol and EFB core-lignophenol were 0.46 and 0.42 , respectively. The ${ }^{1} \mathrm{H}-\mathrm{NMR}$ spectrum of $\mathrm{EFB}$ core-lignophenol was similar to that of hardwood lignophenol (Fig. 3, b). These results show that the core-lignin of EFB lignophenol consists of guaiacyl and syringyl units.

The amount of phenolic hydroxyl group in EFB core-lignophenol was lower than that of EFB lignophenol, while the amount of aliphatic hydroxyl group was higher than that of EFB lignophenol (Table1). The decrease of phenolic hydroxyl group was also confirmed by the differential ionization spectra of EFB lignophenol and EFB core-lignophenol (Fig. 4).

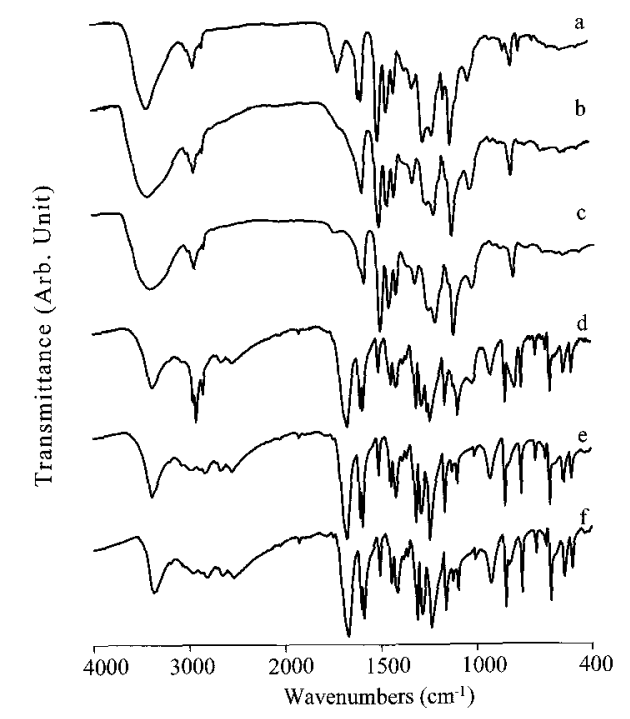

Fig. 1 FT-IR spectra of lignophenols and alkaline hydrolysis products. [a: EFB lignophenol, b: EFB core-lignophenol, c: Hardwood lignophenol, d: Alkaline hydrolysis product from EFB lignophenol, e: Product obtained after recrystallization of alkaline hydrolysis product with water, f: p-Hydroxybenzoic acid. ]
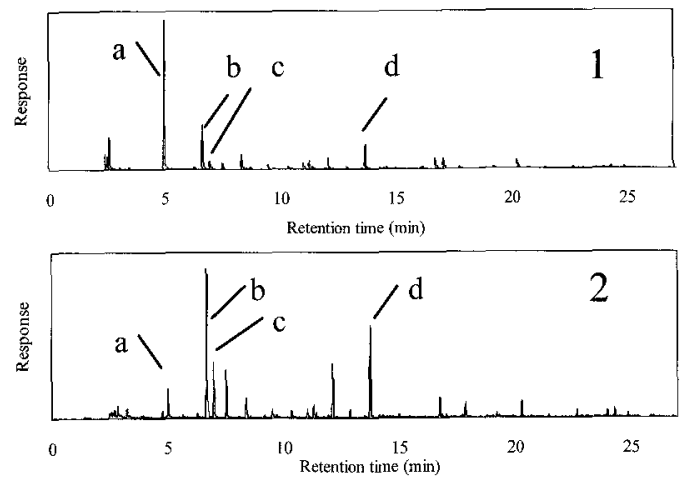

Fig. 2 Pyrograms of EFB lignophenols. [1: EFB lignophenol, 2: EFB core-lignophenol, a: Phenol, b: $p$-Cresol, c: Guaiacol, d: Syringol.]

The average molecular weight $\overline{(\mathrm{M} w)}$ of EFB core-lignophenol was about 4,000 . These results suggest that phenolic carboxylic acid is linked to the side chain of G-S type core lignin through ester linkage, and DP of lignin subunits formed by radical coupling in biosynthesis of EFB lignin is in the range of 13-14.

Table1 Amounts of grafted cresol, average molecular weights and hydroxyl groups of lignophenols

\begin{tabular}{|c|c|c|c|c|c|c|c|c|c|}
\hline \multirow{3}{*}{ Sample } & \multirow{2}{*}{\multicolumn{2}{|c|}{ Grafted cresol contents }} & \multicolumn{4}{|c|}{ Hydroxyl groups } & \multirow{2}{*}{\multicolumn{2}{|c|}{ Iverage molecular weights }} & \multirow[b]{3}{*}{$\overline{\mathrm{M}} w / \overline{\mathrm{M}} \mathrm{n}$} \\
\hline & & & \multicolumn{2}{|c|}{ Phenolic OH } & \multicolumn{2}{|c|}{ Aliphatic $\mathrm{OH}$} & & & \\
\hline & $w t(\%)$ & $\mathrm{mol} / \mathrm{C}_{9}$ & $w t(\%)$ & $\overline{\mathrm{mol} / \mathrm{C}_{9}}$ & $w t(\%)$ & $\overline{\mathrm{mol} / \mathrm{C}_{9}}$ & $\overline{\mathrm{M}} \mathrm{w}$ & $\overline{\mathrm{M}} \mathrm{n}$ & \\
\hline EFB lignophenol & 26.2 & 0.72 & 7.58 & 1.31 & 3.06 & 0.56 & 6184 & 3522 & 1.76 \\
\hline EFB core-lignophenol & 27.8 & 0.78 & 6.45 & 1.13 & 6.87 & 1.20 & 4074 & 2436 & 1.67 \\
\hline
\end{tabular}



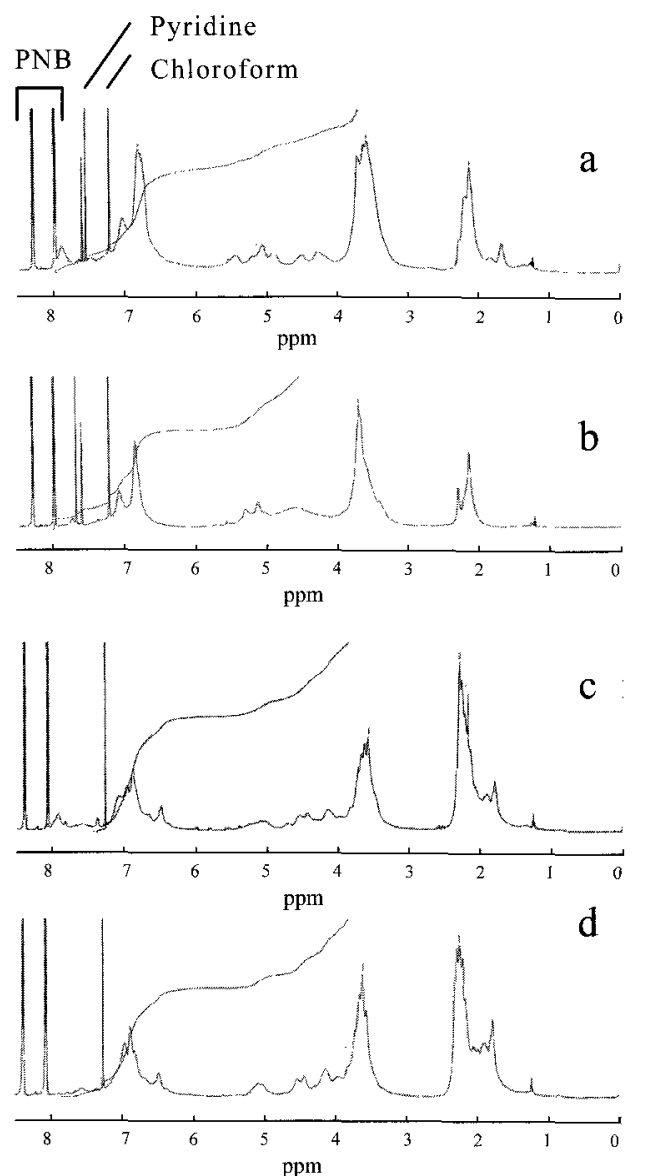

Fig. $3{ }^{1} \mathrm{H}-\mathrm{NMR}$ spectra of lignophenols. [a: EFB lignophenol (original), b: EFB core-lignophenol (original), c: EFB lignophenol (acetylated), d: EFB core-lignophenol (acetylated).]

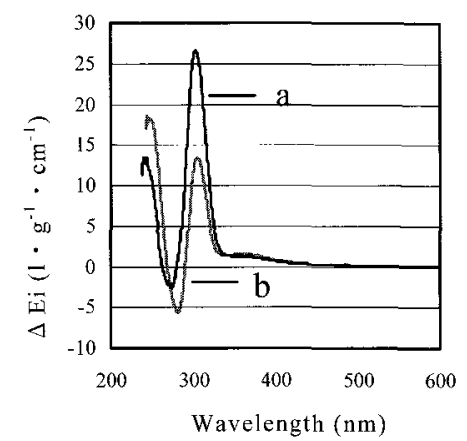

Fig. 4 Ionization differencial spectra of lignophenols. [a: EFB lignophenol, b: EFB core-lignophenol.]
I

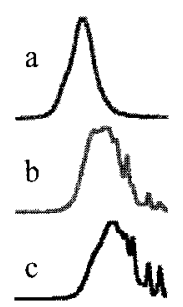

II
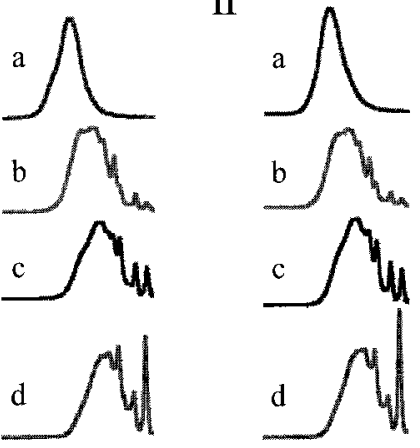

$25 \quad 30 \quad 35$

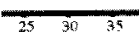

Fig. 5 GPC profiles of lignophenols and 2nd derivatives. [ I : EFB lignophenol, II : EFB core-lignophenol, Treatment temperature; a: Original (untreated), b: $120{ }^{\circ} \mathrm{C}, \mathrm{c}: 140{ }^{\circ} \mathrm{C}, \mathrm{d}$ : $170{ }^{\circ} \mathrm{C}$.]

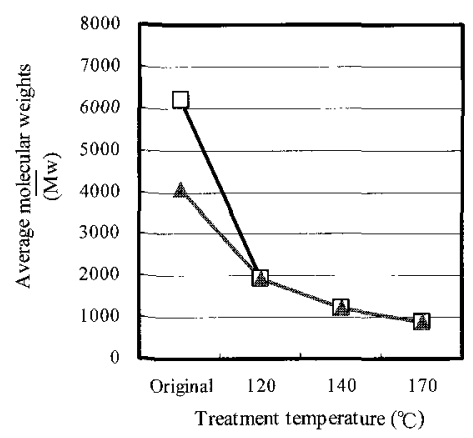

Fig. 6 Average molecular weights of lignophenols and 2nd derivatives. [ $\square$ :EFB lignophenol, $\$$ :EFB core-lignophenol.]

After alkaline treatments at $120^{\circ} \mathrm{C}, 140^{\circ} \mathrm{C}$ and $170^{\circ} \mathrm{C}$ of EFB core-lignophenols, the average molecular weights were decreased. The variation of average molecular weight was similar to that of EFB lignophenol, indicating the presence of 1,1-bis (aryl) propane units working as switching devices [3] (Fig. 5, Fig. 6)

3.2 The structure and frequency of $p$-hydroxyphenyl units

FT-IR spectrum of the alkaline hydrolysis product (Fig. 1, d) has a peak around $1674 \mathrm{~cm}^{-1}$ assigned to conjugated carboxyl groups. Furthermore the product obtained after recrystallization of alkaline hydrolysis product from EFB lignophenol with water was similar to that of $p$-hydroxybenzoic acid, suggesting that main constituent of the alkaline hydrolysis product is p-hydroxybenzoic acid.

Table 2 Yields of $p$-hydroxybenzoic acid, $p$-coumaric acid and ferulic acid.

\begin{tabular}{|c|c|c|c|c|c|c|c|c|c|}
\hline \multirow[b]{2}{*}{ Sample } & \multicolumn{3}{|c|}{$p$-Hydroxybenzoic acid } & \multicolumn{3}{|c|}{$p$-Coumaric acid } & \multicolumn{3}{|c|}{ Feruric acid } \\
\hline & $\begin{array}{l}\text { wt ( } \% \text { of } \\
\text { sample) }\end{array}$ & $\begin{array}{c}\text { wt ( } \% \\
\text { of lignin) }\end{array}$ & $\begin{array}{l}\mathrm{mol} / \mathrm{C}_{9} \\
\text { in lignin }\end{array}$ & $\begin{array}{l}\text { wt ( } \% \text { of } \\
\text { sample) }\end{array}$ & $\begin{array}{c}\text { wt }(\% \text { of } \\
\text { lignin) }\end{array}$ & $\begin{array}{l}\mathrm{mol} / \mathrm{C}_{9} \\
\text { in lignin }\end{array}$ & $\begin{array}{c}\text { wt }(\% \\
\text { sample) }\end{array}$ & $\begin{array}{c}\text { wt ( } \% \\
\text { of lignin) }\end{array}$ & $\begin{array}{l}\mathrm{mol} / \mathrm{C}_{9} \\
\text { in lignin }\end{array}$ \\
\hline EFB meals & 1.8 & 8.8 & 0.15 & 0.05 & 0.25 & 0.004 & trace & trace & trace \\
\hline EFB lignophenol & 9.6 & - & 0.23 & trace & trace & trace & - & - & - \\
\hline
\end{tabular}


The GC/MS chromatograms and mass spectra of alkaline hydrolysis product from EFB lignophenol and EFB meals were shown in Fig. 7 and Fig. 8. The alkaline hydrolysis product from EFB lignophenol contained $p$-hydroxybenzoic acid mostly. This result was also confirmed by FT-IR spectrum of alkaline hydrolysis product from EFB lignophenol after recrystallization (Fig. 1, e). The alkaline hydrolysis product from EFB meals contained $p$-hydroxybenzoic acid and $p$-coumaric acid.
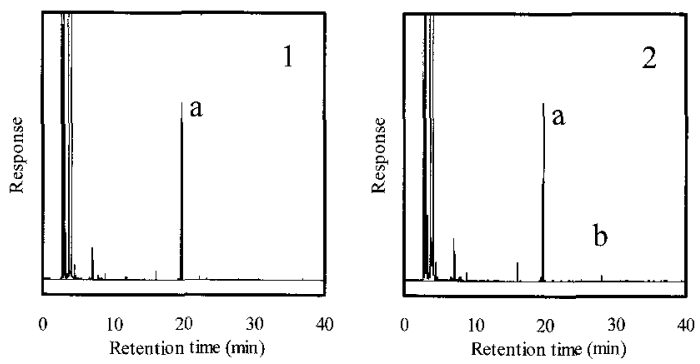

Fig. $7 \mathrm{GC} / \mathrm{MS}$ chromatograms of alkaline hydrolysis product from EFB lignophenol and EFB meals. [1: Alkaline hydrolysis product from EFB lignophenol, 2: Alkaline hydrolysis product from EFB meals, a: $p$-Hydroxybenzoic acid, b: $p$-Coumaric acid]
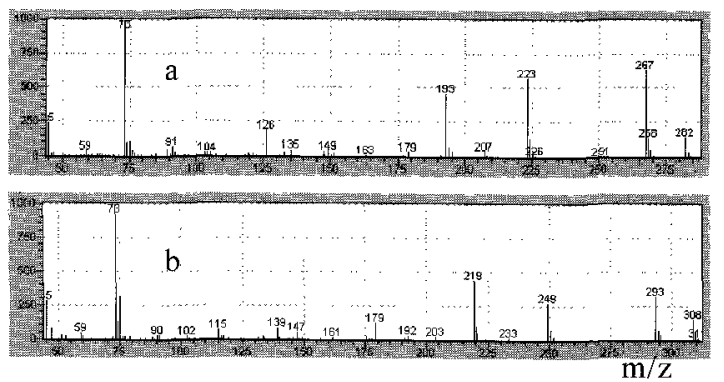

Fig. 8 Mass spectra of main peaks of alkaline hydrolysis products. [a: $p$-Hydroxybenzoic acid (Fig. 7, 1-a), b: p-Coumaric acid(Fig. 7, 2-b).]

The amounts of attached $p$-hydroxybenzoic acid were $9.6 \%$ of EFB lignophenol $\left(0.23 \mathrm{~mol} / \mathrm{C}_{9}\right.$ in EFB lignophenol) and $1.8 \%$ of EFB extractives-free meals $\left(0.15 \mathrm{~mol} / \mathrm{C}_{9}\right.$ in klason lignin). This result shows that ester linkages of $p$-hydroxybenzoic acid are not cleaved during phase-separation treatment because of solvation effect of phenol. EFB lignin was converted to ether insoluble lignophenol (about 70\%) and ether soluble lignophenol (about 30\%) through the phase-separation system. The difference in frequencies of $p$-hydroxybenzoic acid suggests the heterogeneous incorporation of p-hydroxybenzoic acid into lignin (Table2).

3.3 The role of $p$-hydroxyphenyl units in EFB lignin

From the results of TMA and TG, the thermal stabilities of EFB core-lignophenol were higher than those of EFB lignophenol (Fig. 9, Fig. 10). The phase-transition points and $10 \%$ weight loss points of EFB lignophenol and EFB core-lignophenol were $162{ }^{\circ} \mathrm{C}$ and $192^{\circ} \mathrm{C}, 235^{\circ} \mathrm{C}$ and $254^{\circ} \mathrm{C}$, respectively. The pattern of TMA of homogeneous mixture of $\mathrm{EFB}$ core-lignophenol and $p$-hydroxybenzoic acid was similar to that of EFB lignophenol. This result show that $p$-hydroxybenzoic acid works as internal plasticizer.

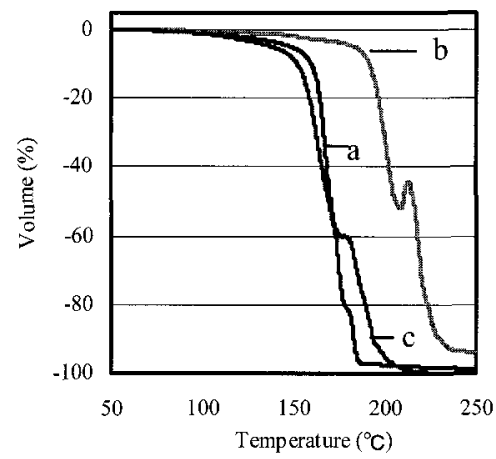

Fig. 9 TMA profiles of lignophenols. [a : EFB lignophenol, b: EFB core-lignophenol, c: Homogeneous mixture of EFB core-lignophenol $(10.3 \mathrm{mg})$ and $p$-Hydroxybenzoic acid (1.08 mg).]

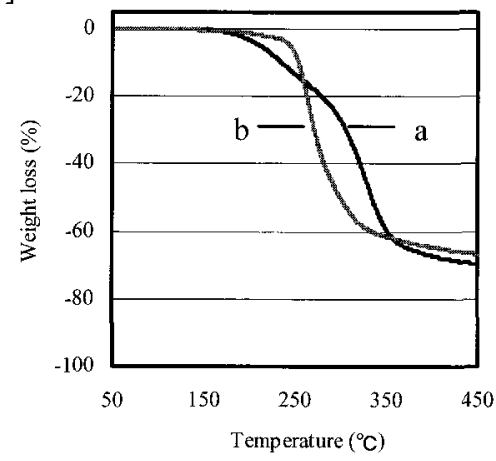

Fig. 10 TG profiles of lignophenols. [a :EFB lignophenol, b:EFB core-lignophenol.]

\section{CONCLUSION}

EFB core-lignin consists of guaiacyl and syringyl units, and carboxyl groups of $p$-hydroxybenzoic acid are linked to aliphatic groups of EFB core-lignin, showing that EFB lignin is classified with grass lignin. Furthermore it is easy to release $p$-hydroxybenzoic acid, showing that EFB lignin is successively structure-controllable. $p$-Hydroxybenzoic acid is valuable as an organic resource, medical supply and phenolic agents for sustainable phase-separation system without petroleum. EFB is superior to woody materials as industrial raw materials.

\section{REFERENCE}

[1]Shinano, T., M. Funaoka, Y. Shirai and M. A. Hassan, Trans. Mater. Res. Soc. J., in press (2008).

[2]Funaoka, M. and I. Abe, Tappi. J., 72, 145-149 (1989)

[3]Funaoka, M., Polym. Int., 47, 277-290 (1998)

[4]Nakamura, Y. and T. Higuchi, Holzforschung, 30, 187-191(1976) 